\title{
Les inventives. Femmes, inventions et brevets en France à la fin du XIX ${ }^{\mathrm{e}}$ siècle
}

Inventress. Women, inventions and patents in France at the end of XIX ${ }^{\text {th }}$ century

\section{Anne Chanteux}

\section{CpenEdition}

\section{Journals}

Édition électronique

URL : http://journals.openedition.org/dht/519

DOI : $10.4000 /$ dht.519

ISSN : 1775-4194

Éditeur :

Centre d'histoire des techniques et de l'environnement du Cnam (CDHTE-Cnam), Société des élèves du CDHTE-Cnam

Édition imprimée

Date de publication : 31 mars 2009

Pagination : 90-97

ISBN : 978-2-95-30779-3-3

ISSN : 0417-8726

Référence électronique

Anne Chanteux, "Les inventives. Femmes, inventions et brevets en France à la fin du XIXe siècle », Documents pour l'histoire des techniques [En ligne], 17 | $7^{\text {er }}$ semestre 2009, mis en ligne le 29 septembre 2010, consulté le 08 septembre 2020. URL : http://journals.openedition.org/dht/519 ; DOI : https:// doi.org/10.4000/dht.519 


\title{
Les inventives. Femmes, inventions et brevets en France à la fin du $X I X{ }^{e}$ siècle
}

\author{
Anne Chanteux \\ Musée des arts et métiers - CDHTE-Cnam
}

\begin{abstract}
RÉSUMÉ
Femme et invention : les deux termes sont rarement associés bien que les brevets déposés par des femmes indiquent leur présence constante dans le paysage de l'invention technique. La littérature anglo-saxonne s'est déjà penchée sur cet épiphénomène, pas la France. Pourtant les registres de I'INPI (Institut national de la propriété industrielle) attestent leur vitalité dans le domaine de l'invention et ce depuis le début des dépôts (1791). L'article s'intéresse à la période fin XIXe-début XXe siècle. Empêchées d'accéder à un enseignement scientifique et technique de qualité, juridiquement inféodées aux hommes, des femmes ont quand même su s'inscrire dans une dynamique créative. Même si nous ne pouvons suivre le parcours de l'invention tout du long, de son origine jusqu'à sa production, l'étude des brevets apporte des éclairages intéressants sur le rôle des femmes dans l'histoire des techniques.
\end{abstract}

Résumés et mots clés en anglais sont regroupés en fin de volume, accompagnés des mots clés français

$C_{m e n s}$ et article souhaite rendre compte d'une activité méconnue dans l'histoire des techniques comme dans celle des femmes : la littérature française propre à ces deux disciplines n'évoque jamais le rôle que des femmes ont pu jouer dans le domaine des techniques et de l'invention'. L'étude d'un corpus de brevets relevé dans un journal féministe du XIX siècle, La Fronde, permet de faire un pont entre les femmes et les techniques. Ce journal a publié durant trois années des listes de brevets déposés par des femmes, listes présentées comme l'exemple de l'implication féminine dans toutes les branches de l'activité et du savoir. Le journal ne s'en tenait pas à la publication des listes puisqu'il se faisait l'écho de l'actualité scientifique et technique avec une grande régularité. Ses journalistes ont donné une nouvelle image publique de la femme en investissant des sphères conventionnellement masculines (parlement, tribunaux, bourse, préfectures, conseils municipaux, .... $)^{2}$. L'étude de ce

1 Article extrait d'un mémoire de DEA soutenu au CDHTE en 2006 sous la direction de Liliane Pérez.

2 Séverine Auffret, Séverine, Université populaire de Caen, séminaire d'idées féministes, cours du 24 mars 2005 [en li- journal a marqué le point de départ de cette analyse : femmes et invention à la fin du XIXe siècle et au début du XXe siècle.

En retraçant le contexte éducationnel, on s'aperçoit en effet qu'il est peu propice malgré les grands progrès que marque cette époque - loi Camille Sée de 1880 organisant l'enseignement secondaire féminin, réforme Bérard de 1924 assimilant les deux enseignements, pour filles et garçons. Le sexe féminin obtient ainsi un accès à l'éducation et notamment à l'enseignement technique. II demeure cependant éloigné des savoirs susceptibles de produire des inventions liées aux développements techniques importants de cette fin de XIXe siècle. Pourtant, des femmes ont produit des inventions et les ont brevetées. Les répertoires disponibles à l'Institut national de la propriété industrielle (INPI) permettent de constater l'étendue des domaines couverts par ces brevets, qui ne se contentent pas d'améliorer la vie domestique.

gne]. [réf. du 10 février 2009]. http://pagesperso-orange.fr/ michel.onfray/Auffret24mars05.pdf 


\section{Femmes et inventions : le paysage}

État de la question

Des universitaires féministes américaines et canadiennes se sont penchées sur les relations entre femmes et techniques. II s'agit le plus souvent de travaux de sociologues sur l'appropriation des techniques par les femmes, dans la vie quotidienne ou professionnelle, ou des rapports sociaux entre hommes et femmes: "Ce qui a longtemps été au centre de l'intérêt des féministes critiques de la technique, ce n'est pas tant la technique que les rapports de domination entre les hommes et les femmes. Longtemps les techniques ne sont pas étudiées en tant que telles $1{ }^{3}$. En France, quelques chercheuses sortent du seul cadre de la sociologie, de l'histoire des femmes ou de l'histoire des sciences et des techniques pour tenter des ponts inter disciplines et élargir ainsi le débat ${ }^{4}$. Mais aucune étude française ne s'est intéressée aux femmes inventrices ${ }^{5}$. Seule la littérature anglo-saxonne les a prises en compte.

«L'histoire des inventions représente les inventeurs de l'Antiquité, du Moyen Age et de la Renaissance comme étant invariablement des hommes. La question de savoir quelle part ont ou n'ont pas jouée les femmes dans l'invention technique est une question épineuse. Les femmes ont presque toujours été « cachées de l'histoire» quand les historiens étaient des hommes $1^{6}$.

S'appuyant sur les travaux d'Autumn Stanley, de Cynthia Cockburn, de Judy Wajcman, entre autres, des chercheurs ont montré que, si les femmes semblent invisibles dans le domaine technique, elles occupent néanmoins le terrain domestique avec de

3 Danièle Chabaud-Rychter, Delphine Gardey dir., L'engendrement des choses: des hommes, des femmes et des techniques, Paris, Ed. des archives contemporaines, 2002, p. 32. 4 Voir les ouvrages coordonnés par Danièle Chabaud-Rychter, Delphine Gardey, par exemple.

5 Les ouvrages les plus récents sur le sujet sont titrés Femmes inventeurs couronnés par l'Organisation mondiale de la propriété industrielle (1991) ou Les femmes inventeurs existent, je les ai rencontrées (1986)... faisant fi, entre autres de l'accord de l'adjectif avec le nom, ce qui, en soit, est significatif. 6 « The history of invention represents the inventors of antiquity, the Middle Ages and the Renaissance as invariably male. The question of what part women did or did not play in technological invention is a knotty one. Women have almost always been "hidden from history" when the historians were men ", Cynthia Cockburn, "Technology, production and power "1, dans Gill Kirkup, Laurie Smith Keller éd., Inventing women : science, technology and gender, Oxford, Polity press, the Open university, 2004, p. 199. réelles pratiques techniques et développent depuis toujours des innovations dans le champ qui leur est octroyé, à savoir celui de la maison, de l'éducation, de l'alimentation et de la protection (habillement) des leurs. S'attachant à l'usage des techniques dans la vie quotidienne, ils ont voulu prouver que les femmes, comme les hommes, inventent leurs propres outils et sont actives face aux innovations. Au XIX' siècle déjà, Virginie Penny, écrivaine américaine citée par la Revue scientifique des femmes, faisait remarquer avec bon sens :

"I'homme qui exclut la femme de toute instruction technique lui reproche de ne rien inventer, mais il oublie un fait très important, c'est que la société, en ne lui laissant que l'aiguille, les ciseaux, le fuseau et la quenouille, restreint la sphère des occupations de la femme. Or, la tradition nous dit, justement, que tous ces objets ont été inventés par des femmes. Si elles les ont inventés dès qu'elles en ont senti la nécessité, qui les aurait empêchées d'inventer d'autres outils si elles avaient eu d'autres occupations ? $11^{7}$.

Cette citation montre que la réflexion sur les femmes et l'invention n'est pas neuve. Elle s'inscrit dans l'émergence des mouvements féministes au XIXe siècle.

Une deuxième voie des recherches sur le sujet conduit à retracer des " parcours individuels de femmes inventeurs, détentrices de brevets et ingénieurs, dans leurs combats pour se tailler une place dans un monde traditionnellement défini comme masculin $)^{8}$. C'est une deuxième lecture du livre d'Autumn Stanley, par exemple, portraits rapidement esquissés de femmes inventrices ${ }^{9}$. Selon le degré de notoriété des femmes et les informations disponibles, des notices biographiques plus ou moins étoffées retracent leur parcours familial et professionnel, le contexte social dans lequel elles ont vécu et comment elles ont pu innover dans un secteur donné.

Une troisième voie consiste à s'intéresser aux inventions elles-mêmes. Les brevets déposés et exploités par les femmes, témoignant d'un dynamisme féminin dans le domaine de l'invention et de l'innovation, ont fait l'objet d'études circonstanciées dont quatre

7 Revue scientifique des femmes, $n^{\circ} 5$, novembre 1888. 8 Nina E. Lerman, Arwen Palmer Mohun, Ruth Oldenziel, "L'histoire des techniques et la question du genre : état des travaux et perspectives pour l'avenir "), dans D. ChabaudRychter, D. Gardey dir., op. cit. note 3, p. 79.

9 Autumn Stanley. Mothers and daughters of invention : notes for a revised history of technology, Lanham, Md. \& London, The Scarecrow press, inc., 1993. 


\section{Les inventives. Femmes, inventions et brevets en France}

exemples (deux sont anglais, les deux autres sont américains) sont présentés ci-dessous.

\section{Études anglo-saxonnes}

Contrairement aux ouvrages français sur l'innovation et les brevets, un ouvrage sur les brevets d'inventions anglais, British Patents of inventions, mentionne à deux reprises le cas des femmes inventrices ${ }^{10}$ : de 1894 à 1965, le bureau des brevets anglais (Patent Office) relevait le nombre de brevets demandés par des femmes dans son rapport annuel, ainsi que les thèmes les plus fréquents. Les conditions d'obtention sont étudiées dans cet ouvrage, ce qui permet de relativiser la présence ou l'absence des femmes parmi les déposants : en effet, jusqu'au Married Women's Property Act, en 1882, les femmes ne pouvaient disposer de leurs biens, donc jouir de l'exploitation de leurs brevets, sauf à le faire avec l'accord de leur mari. Une autre étude, consacrée aux femmes inventrices, a exploité les brevets féminins anglais ${ }^{11}$ : recensement nom par nom puisque, comme l'établissement français, le Patent Office n'autorise pas la recherche sexuée (hormis la parenthèse des années citées ci-dessus). L'auteur, Deborah Jaffe, s'est intéressée au nombre de brevets, aux thématiques puis a listé les inventions féminines. Elle signale : «Les inventions réalisées par ma sélection de femmes couvrent presque tous les aspects de la vie et montrent combien elles étaient habiles pour résoudre des problèmes et examiner les choses dans une vaste perspective pour le bien de la société ou sur une plus petite échelle, plus intime et personnelle ${ }^{12}{ }^{12}$. En plus de l'étude statistique qui révèle que, depuis le premier brevet déposé au nom d'une femme anglaise en 1637, les inventrices ont régulièrement été présentes dans le paysage des Patents, les thèmes de leurs inventions sont abordés. II en ressort qu'outre la variété des domaines, les inventions sont étroitement liées au contexte socio-économique de leur époque, ce qui montre que l'implication des femmes n'est pas limitée à la vie de famille et qu'elles sont présentes, quoique peu visibles, dans toutes les branches de l'activité humaine.

10 Stephen van Dulken, British patents of invention, 16171977, London, The British library, 1999, pp.84, 90.

11 Deborah Jaffe, Ingenius women: from tincture of saffron to flying machines, Stroud, Sutton, 2003.

12 « The inventions by my selected women have covered almost every aspect of life and shown how adept they were at problem-solving and examining things in a broad perspective for the good of society or on a small, more intimate and personal scale», Deborah Jaffe, "Ingenius women », The CIPA journal (Chartered Institute of patent agents), 33-5, mai 2004, pp. 285-290.
Un ouvrage américain récent, celui de Zorina B. Khan, comporte deux chapitres importants qui concernent les dépôts de brevets féminins aux États-Unis au XIXe siècle. Le premier chapitre porte sur les inventions brevetées et leurs applications commerciales (étude statistique et analyse par thèmes, par origines professionnelles, géographiques, sociales, ...), le second met en relation les lois concernant les femmes et les effets sur leur production de brevets et leur rôle dans l'innovation économique ${ }^{13}$. "Le manque d'analyse quantitative est partiellement dû à la pénurie de données pertinentes à une époque où les femmes étaient rendues "invisibles" par les conventions légales et sociales $11^{14}$. Une autre étude, partielle, avait été menée dès 1979 , portant notamment sur les femmes exposant lors de I'Exposition internationale de Philadelphie en $1876^{15}$. L'auteur avait produit des chiffres intéressants et montrait que, dès 1891, les femmes américaines ont revendiqué leur appartenance au monde de l'invention en créant le journal The Woman inventor, en demandant et en obtenant de la part de l'institution des listes de brevets déposés par les femmes.

\section{Où en sommes-nous du côté français ?}

II ressort de ce tour d'horizon que les chercheurs anglo-saxons ont traité le sujet femmes et inventions par le biais des brevets : « [...] les archives des brevets, quoique imparfaites, fournissent la source la plus objective ef la moins spéculative de connaissance au sujet des femmes et de l'invention ${ }^{16}{ }^{16}$. II n'existe pas d'étude des brevets pris par des femmes en France, pas plus que d'interrogations sur leur implication dans le champ de l'invention. Aborder le sujet par une analyse statistique des dépôts de brevets peut éclairer un pan de l'histoire des techniques laissé en friche.

La France était très en retrait des avancées juridiques anglo-saxonnes concernant les femmes $^{17}$. Y aurait-il un lien entre la reconnaissance de

13 Zorina B Khan, The democratization of invention : patents and copyrights in American economic development, 17901920, Cambridge University Press, 2005.

14 « The lack of quantitative analysis is partly due to the paucity of relevant data in an era when women were rendered "invisible" by legal and social conventions », ibid., p. 132. 15 Deborah J. Warner, "Women inventors at the Centennial ", dans Martha Moore Trescott dir., Dynamos and virgins revisited: women and technological change in history, Metuchen, NJ \& London, The Scarecrow press inc., 1979.

16 ॥ [...] patent records, although imperfect, still provide the best objective and unspeculative source of knowledge about women in invention ॥, Z. B. Khan, The democratization of invention, op. cit. note 13, p. 133.

17 Nicole Arnaud-Duc, "Les contradictions du droit ", dans Geneviève Fraisse, Michelle Perrot dir., Histoire des femmes 
la femme en tant que citoyenne et sa participation à l'innovation technique ? L'histoire montre que l'Angleterre et les États-Unis ont été des précurseurs en matière de droits favorables aux femmes : le Married women's property act (voté en 1848 aux États-Unis, en 1882 en Grande-Bretagne), ainsi qu'un ensemble de lois, donnent aux femmes mariées le contrôle de leurs biens et de leurs gains ${ }^{18}$. La France laisse durant tout le XIX $X^{e}$ siècle (et une grande partie du XXe siècle) la femme sous la tutelle juridique de l'homme, père ou mari. Cette seule donnée peut éclairer une étude des brevets : I'intérêt de breveter tient à la reconnaissance de l'invention et à son exploitation ; si l'inventrice ne peut pas bénéficier de sa découverte, à quoi bon la rendre publique ? Si des femmes ont pu prendre des brevets en leurs noms, il faut donc qu'elles aient bénéficié d'une certaine autonomie, de fait (veuvage) ou accordée par leur tutelle masculine.

Pourquoi et comment déposer un brevet? "Le brevet d'invention est un titre délivré par le gouvernement pour conférer à un inventeur le droit exclusif et temporaire d'exploiter l'objet de sa découverte, sous certaines conditions $1{ }^{19}$. Le dépôt d'un brevet suppose que l'inventeur souhaite protéger son invention et la produire ou la faire produire. D'ailleurs la loi l'oblige à mettre en exploitation son invention sous peine d'être déchu de tous ses droits (article 32 de la loi du 5 juillet 1844). La démarche de dépôt d'un brevet suit des règles strictes (description de l'invention, dessin) et n'est pas gratuite : une taxe est demandée pour le dépôt et, lors de l'attribution du brevet, une somme dépendant de la durée de protection choisie est perçue par l'état. Des annuités doivent être versées par l'inventeur sous peine de déchéance de ses droits. Dans ces conditions, il faut être motivé, disposer immédiatement d'argent (plus ou moins selon qu'on dépose seul ou qu'on fait appel à un agent) et être prêt à investir commercialement. Conditions peu simples à réunir à une époque où le code Napoléon place la femme en position de tutelle juridique. La France, contrairement à l'Allemagne et aux ÉtatsUnis, n'examine pas la demande avant d'octroyer un brevet : les brevets sont "SGDG ॥ (sans garantie du gouvernement) mais l'inventeur n'ayant pas créé ou apporté de perfectionnement significatif s'expose à la nullité de son dépôt et à la déchéance de ses droits

en Occident. Tome 4, le XIXe siècle, Paris, Plon, 1991.

18 Z. B. Khan, The democratization of invention, op. cit. note 13 et D. J. Warner, "Women inventors at the Centennial ", op. cit. note 15

19 Eugène-Oscar Lami, Dictionnaire encyclopédique et biographique de l'industrie et des arts industriels. 1, A-B, Paris, Librairie des dictionnaires, 1881, p. 941. s'il est avéré que la condition de nouveauté n'est pas respectée.

Le fonctionnement du système des brevets dessine le portrait de l'inventrice : relativement autonome puisqu'elle peut disposer d'argent et être représentante légale, possédant un savoir technique puisqu'elle connaît la valeur de son invention et la juge digne d'un brevet, active socialement puisqu'elle intègre à son savoir celui des réseaux de protection et de valorisation de l'invention. Reste à affiner ce portrait pour comprendre comment et pourquoi un petit pourcentage de femmes brevette et ce que devient leur invention.

\section{Analyse des brevets}

Le corpus utilisé provient du dépouillement du quotidien La Fronde. Le journal commence la publication des listes de brevets en 1900 (première liste le 18 mai) : " grâce à l'obligeance de MM. Marillier et Robelet (office international pour l'obtention des brevets d'inventions en France et à l'étranger, 42 boulevard Bonne Nouvelle, Paris), nous pourrons chaque mois faire connaître à nos lecteurs les brevets délivrés aux femmes $1{ }^{20}$. L'office des ingénieurs civils Marillier et Robelet a adressé, le 27 avril 1900, une lettre à Marguerite Durand, directrice du journal, pour proposer l'envoi, tous les quinze jours ou tous les mois, de listes recensant «les derniers brevets français pris par des femmes $"{ }^{21}$. Ce courrier est assez intéressant pour être cité en majeure partie :

" II arrive assez fréquemment en France que des brevets d'invention sont pris par des femmes dans toutes les industries, mais plus particulièrement dans celles qui les intéressent le plus directement, c'est-à-dire les vêtements, l'hygiène, l'alimentation, etc.

Nous venons vous demander si vous pensez qu'il serait intéressant pour vos lectrices d'être mises au courant de l'existence de ces brevets. En tout cas, cette communication ne pourrait, à notre avis, qu'être utile à l'œuvre d'émancipation que vous poursuivez $1{ }^{22}$.

Jusqu'à la création de l'Office national de la propriété industrielle (ONPI) en 1901, qui réunira dans un même lieu les catalogues et les publications de brevets, il est difficile de consulter et de reproduire les

20 La Fronde, vendredi 18 mai 1900.

21 Lettre de MM. Marillier et Robelet à Mme Marguerite Durand, datée du 27 avril 1900. Bibliothèque Marguerite Durand, dossier documentaire sur les femmes et les inventions, DOS 600 INV. 22 lbid. 


\section{Les inventives. Femmes, inventions et brevets en France}

brevets $^{23}$. Les agences d'ingénieurs ont la charge de diffuser l'information technique : " Avant 1902, date à laquelle l'État prend enfin ses responsabilités en matière de publication de brevets d'invention, c'est en effet aux agents d'inventions qu'échoit la tâche de publier, partiellement, les brevets d'invention récents ${ }^{24}$. De plus, la recherche par genre n'est pas possible. L'office de MM. Marillier et Robelet propose donc un service précieux en mettant à disposition des listes comprenant le numéro de brevet, la date de dépôt, le nom de la déposante, l'intitulé du brevet.

En échange de ce travail, les ingénieurs demandent « ... simplement d'en indiquer la provenance au bas de chaque liste que vous publierez $\|{ }^{25}$. II semble qu'il y ait eu émulation car le dossier documentaire de la Bibliothèque Marguerite Durand contient deux lettres datées de 1925, provenant de deux cabinets pour l'obtention des brevets : $\vee$. Prévost et T. Durand, et l'office Picard, proposant tous deux le même service.

De ce premier courrier daté de 1900, on peut déduire que la publication des brevets ne vient pas des journalistes de La Fronde mais d'une proposition extérieure (et masculine !). Le journal tirant à l'époque environ à 14600 exemplaires (chiffres de 1899) ${ }^{26}$, on peut se demander quel bénéfice pouvait espérer l'office de MM. Marillier et Robelet en effectuant, tous les 15 jours ou tous les mois, le recensement des brevets déposés par des femmes. Quelles retombées pouvait-il attendre de la publication de son nom dans un journal à si petit tirage et à lectorat majoritairement féminin? Est-ce la notoriété du journal, de ses collaboratrices qui a incité les directeurs à ce surcroît de travail ? Ontils fait une démarche semblable auprès des autres journaux féministes ? On peut le supposer puisque le journal La Femme de l'avenir publie également des listes en $1901^{27}$. Le petit pourcentage que représentaient les brevets déposés par des femmes suffit-il à justifier cette implication ou ont-ils pensé, plus prosaïquement, que les femmes ayant des époux, ceux-ci pouvaient

23 Gérard Emptoz, Valérie Marchal, Aux sources de la propriété industrielle : guide des archives de I'INPI, Paris, INPI, 2002, p. 52. 24 Gabriel Galvez-Béhar, "Des médiateurs au sein du système d'innovation : les agents de brevets en France, 18701918 ॥, dans Marie-Sophie Corcy, Christiane DemeulenaereDouyère, Liliane Hilaire-Pérez dir., Les Archives de l'invention : écrits, objets et images de l'activité inventive, des origines à nos jours, Toulouse, CNRS, Méridiennes, coll. Histoire \& techniques, 2007, pp. 437-447, p. 441.

25 Lettre de MM. Marillier et Robelet à Mme Marguerite Durand, op. cit.

26 Jean Rabaud, Marguerite Durand (1864-1936) : «La Fronde »féministe ou «Le Temps » en jupons, Paris, l'Harmattan, 1996, p. 79. 27 En 1901, ce bimensuel très militant édite des listes de brevets pris par des femmes, sans commentaire. également être de futurs clients ? Nous n'avons pas la réponse de Marguerite Durand à ce premier courrier mais elle figure implicitement à travers la publication régulière de ces listes dans La Fronde.

\section{Statistiques}

En se référant aux statistiques de brevets déposés ${ }^{28}$, on peut calculer le pourcentage de brevets féminins cités dans La Fronde, sachant que les listes publiées dans le quotidien ne sont pas exhaustives ${ }^{29}$.

\begin{tabular}{|c|c|c|c|}
\hline & $\begin{array}{c}\text { nombre de brevets dé- } \\
\text { posés par des femmes } \\
\text { et cités dans La Fronde }\end{array}$ & $\begin{array}{c}\text { nombretotaldebrevets } \\
\text { déposés (source: INPI) }\end{array}$ & $\%$ \\
\hline 1900 & 119 & 12400 & $0,95 \%$ \\
\hline 1901 & 136 & 12103 & $1,12 \%$ \\
\hline 1902 & 102 & 12026 & $0,84 \%$ \\
\hline
\end{tabular}

Fig. 1 - Les brevets des femmes dans La Fronde (l'année 1899 a été omise, le nombre recensé dans le joumal ne portant que sur un semestre)

Aux États-Unis, les femmes obtenaient 303 brevets en 1893, soit 1,5\% du total des brevets ${ }^{30}$. En 1898, il y eut en Angleterre 638 brevets pris par des femmes sur un total de 27639 dépôts ${ }^{31}$. Si on laisse de côté l'écart entre les deux pays lié à la tradition en matière de dépôt de brevets, plus ancienne en Angleterre qu'en France ${ }^{32}$, le ratio anglais est tout de même nettement plus élevé : $2,30 \%$, soit plus du double de brevets déposés par des femmes. II s'agit là d'une année exceptionnelle : «le pourcentage ne s'élève pas au-dessus de $2 \%$ après 1912 ou au-dessus de $1 \%$ après $1938 \|{ }^{33}$. Globalement, le pourcentage français semble plus faible. Le dynamisme des Anglo-saxonnes en matière d'inventions peut s'expliquer par leur plus grande autonomie légale et leurs actions pionnières en matière de féminisme.

Les brevets sont listés selon la classification du bureau de la propriété industrielle en usage entre 1884 et 1901 (elle sera grandement modifiée par la suite). La

28 G. Emptoz, V. Marchal, op. cit. note 23, pp. 214, 216. 29 La comparaison de ces listes avec le bulletin officiel de la propriété industrielle et commerciale (BOPI) à l'INPI fait apparaître des brevets supplémentaires, non recensés par le cabinet. La recherche n'ayant pas été systématique, on ne peut évaluer la différence entre la réalité et le recensement publié dans La Fronde. Ce travail sera à mener.

30 D. J Warner, "Women inventors at the Centennial », op. cit. note 15, p. 104.

31 D. Jaffe, « Ingenius women », op. cit. note 12, p. 285.

32 Liliane Hilaire-Pérez. L'invention technique au siècle des Lumières, Paris, Albin Michel, 2000.

33 " The percentage did not rise above $2 \%$ after 1912 or above $1 \%$ after 1938 ॥, S. van Dulken, op. cit. note 10, p. 84. 
répartition des brevets selon cette classification est la suivante, par nombre décroissant de brevets :

\begin{tabular}{|c|c|c|c|}
\hline Classe & Intitulé & $\begin{array}{l}\text { nombre } \\
\text { de brevets }\end{array}$ & $\begin{array}{c}\% \text { par } \\
\text { rapport au } \\
\text { nombre total } \\
(414)\end{array}$ \\
\hline $\mathrm{XVI}$ & Habillement & 93 & 22,46 \\
\hline IX & $\begin{array}{l}\text { Matériel de l'économie } \\
\text { domestique }\end{array}$ & 52 & 12,56 \\
\hline v & Machines & 40 & 9,66 \\
\hline$x x$ & $\begin{array}{l}\text { Articles de Paris et petites } \\
\text { industries }\end{array}$ & 29 & 7 \\
\hline 1 & Agriculture & 27 & 6,52 \\
\hline$x v$ & $\begin{array}{l}\text { Eclairage, chauffage et } \\
\text { réfrigération }\end{array}$ & 23 & 5,55 \\
\hline$x$ & Carrosserie & 22 & 5,31 \\
\hline XIV & Arts chimiques & 22 & 5,31 \\
\hline XIX & $\begin{array}{l}\text { Chirurgie, médecine, } \\
\text { hygiène }\end{array}$ & 18 & 4,34 \\
\hline IV & Arts textiles & 16 & 3,86 \\
\hline XVII & Arts industriels & 15 & 3,62 \\
\hline XVIII & Papeterie & 14 & 3,38 \\
\hline VII & Travaux de construction & 10 & 2,41 \\
\hline XII & Instruments de précision & 8 & 1,93 \\
\hline III & Chemins de fer et tramways & 7 & 1,69 \\
\hline VIII & Mines et métallurgie & 7 & 1,69 \\
\hline II & Hydraulique & 6 & 1,44 \\
\hline VI & Marine et navigation & 2 & 0,48 \\
\hline $\mathbf{X I}$ & Arquebuserie et artillerie & 2 & 0,48 \\
\hline XIII & Céramique & 1 & 0,24 \\
\hline
\end{tabular}

Fig. 2 - Les brevets obtenus par des femmes à la fin du XIXe siècle

Il est intéressant de comparer ces chiffres avec ceux donnés pour la même période par Gabriel Galvez-Behar :

\begin{tabular}{|l|c|c|c|}
\hline & 1895 & 1900 & $1880-1900$ \\
\hline \multicolumn{1}{|c|}{ Total } & 100 & 100 & 100 \\
\hline $\begin{array}{l}\text { Economie domestique et } \\
\text { consommation courante }\end{array}$ & 21,52 & 22,07 & 21,36 \\
\hline $\begin{array}{l}\text { Machines et instruments de } \\
\text { précision }\end{array}$ & 17,49 & 19,69 & 17,51 \\
\hline Agriculture et alimentation & 9,8 & 7,74 & 10,5 \\
\hline Textile et habillement & 8,81 & 7,53 & 9,5 \\
\hline Transports sur route & 10,62 & 7,94 & 7,43 \\
\hline Electricité et téléphonie & 6,49 & 6,24 & 6,25 \\
\hline Chemins de fer et tramways & 5,33 & 6,43 & 5,69 \\
\hline Chimie & 4,91 & 5,1 & 5,28 \\
\hline Travaux, construction, bâtiment & 4,1 & 4,73 & 5,11 \\
\hline Articles de bureau & 2,78 & 2,97 & 2,77 \\
\hline Mines et métallurgie & 2,04 & 2,22 & 2,42 \\
\hline Armement & 2,12 & 1,84 & 2,3 \\
\hline Santé & 1,85 & 3,36 & 2,01 \\
\hline Transport maritime et fluvial & 2,14 & 2,13 & 1,86 \\
\hline
\end{tabular}

Fig. 3 - Répartition des brevets et certificats délivrés par types d'industrie (extraits) ${ }^{34}$

34 Gabriel Galvez-Behar, "Pour la Fortune et pour la Gloire" :
On constate que les répartitions de brevets selon les thèmes sont sensiblement les mêmes (les thématiques de Gabriel Galvez-Behar résultent d'une classification propre établie pour les besoins de sa thèse à partir des différentes classifications du bureau de la propriété industrielle en usage dans les années étudiées). Les femmes inscrivent leur activité inventive dans les secteurs en développement de l'économie.

Dans le tableau thématique des brevets déposés par les femmes, si les deux premières classes (correspondant au classement thématique des brevets) sont représentatives de ce qu'on attend traditionnellement de l'activité inventive des femmes (habillement et matériel de l'économie domestique), la troisième est plus surprenante. La classe Machines comporte 9 sous-classes presque toutes représentées dans les brevets :

\begin{tabular}{|c|c|c|c|c|c|c|}
\hline v & MACHINES & 总 & $\stackrel{0}{\varrho}$ & ¿ & ָั & $\begin{array}{l}\text { sous-total } \\
\text { par sous- } \\
\text { classes }\end{array}$ \\
\hline V-1 & machines à vapeur & 0 & 1 & 1 & 0 & 2 \\
\hline$\vee-2$ & Chaudières & 1 & 0 & 0 & 1 & 2 \\
\hline$\vee-3$ & Organes & 1 & 0 & 2 & 2 & 5 \\
\hline$\vee-4$ & $\begin{array}{l}\text { Machines-outils pour le } \\
\text { travail des métaux et } \\
\text { des bois }\end{array}$ & 3 & 2 & 0 & 1 & 6 \\
\hline$\vee-5$ & Machines diverses & 1 & 3 & 2 & 0 & 6 \\
\hline$\vee-6$ & Manœuvre des fardeaux & 0 & 0 & 0 & 1 & 1 \\
\hline$\vee-7$ & Machines à coudre & 0 & 2 & 2 & 1 & 5 \\
\hline$\vee-8$ & Moteurs divers & 1 & 6 & 4 & 2 & 13 \\
\hline \multirow[t]{2}{*}{$\vee-9$} & $\begin{array}{l}\text { Machines servant à la fa- } \\
\text { brication des chaussures }\end{array}$ & 0 & 0 & 0 & 0 & 0 \\
\hline & sous-total par année & 7 & 14 & 11 & 8 & 40 \\
\hline
\end{tabular}

Fig. 4-Les brevets de la classe «machines » obtenus par des femmes

La sous-classe machines à coudre n'est pas la plus riche. Perfectionnement aux machines à travailler les tôles ; nouveau système de moteur à vapeur ou à air comprimé et servant à actionner des forets ou autres outils similaires; perfectionnement à la fermeture des vases, récipients, appareils, chaudières, etc. ; carburateur pour moteurs à pétrole et similaires, tels sont les intitulés de quelques uns des brevets déposés en classe V-4, V-5 et V-8. II est étonnant de voir des machines, ou des perfectionnements apportées aux machines, inventés par des femmes alors que les secteurs de l'industrie qui leur sont plus particulièrement reconnus comportent peu de dépôts de brevets. Dans les Arts

inventeurs, propriété industrielle et organisation de l'invention en France, 1870-1922, thèse de doctorat d'histoire, Jean-Pierre Hirsch dir., Université Lille-Ill-Charles de Gaulle, Lille, 2004, p. 149. 


\section{Les inventives. Femmes, inventions et brevets en France}

textiles, par exemple, les sous-classes passementerie, tricots, tulles, dentelles et filets, broderies ne mentionnent que 3 brevets sur les 4 années étudiées. Plus surprenant peut-être, alors que les arts appliqués leur sont ouverts, les femmes inventrices répertoriées n'ont déposé qu'un brevet (sous-classe verrerie) dans la classe Céramique. Les Arts industriels, parmi lesquels figurent la peinture, dessin, gravure et sculpture, et la musique ne représentent que 15 brevets (dont aucun pour la peinture et 5 pour la musique, arts d'agrément pourtant couramment pratiqués par la classe bourgeoise).

Ce coup d'œil rapide sur les thèmes des brevets pris par des femmes fait ressortir une variété beaucoup plus grande que prévu. Les auteurs, britanniques et américaines, qui ont étudié les brevets féminins de leur pays respectif annoncent dès l'introduction de leur étude que les femmes inventent principalement dans le champ domestique ${ }^{35}$. Cela est vrai aussi des femmes françaises, pour autant que le petit chiffre pris pour l'étude soit représentatif. "L'économie domestique était devenue, au cours du XIXe siècle, une discipline autonome, pratiquée essentiellement par des femmes $11^{36}$. II n'en reste pas moins qu'elles brevettent dans toutes les classes de l'Office pour la propriété industrielle, classes qui représentent l'état de l'industrie à leur époque. Moins d'un quart des brevets français étudiés relève de la classe Habillement, soit d'un secteur identifié comme relevant du domaine féminin, ce qui laisse une part importante aux classes moins spécifiquement connotées. Les femmes ne produisent donc pas seulement là où on les attend. Cette observation, reproduite à plus grande échelle, peut se révéler intéressante car elle fait naître diverses questions sur les petites ou grandes inventions : quelle est la valeur des inventions les unes par rapport aux autres? Existe-t-il une hiérarchie dans l'invention qui, en dehors de toute considération économique, expliquerait le côté invisible de la femme inventrice ? Une autre question importante tient à l'éducation accordée aux filles : malgré l'ouverture progressive des établissements scolaires à la gent féminine, l'accès possible aux cours techniques pour les salariées et l'offre large de conférences gratuites accessibles à tous les publics (notamment au Conservatoire national des arts et métiers), l'enseignement des filles et des garçons n'est pas le même.

Zorina $B$. Khan explique que les féministes américaines du XIXe siècle voulaient prouver qu'elles pro-

35 Z. B. Khan, The democratization of invention, op. cit. note 13 ; D. J. Warner, "Women inventors at the Centennial", op. cit. note 15 ; D. Jaffe, Ingenius women, op. cit. note 11.

36 François Caron, Les deux révolutions industrielles du XXe siècle, Paris, Albin Michel, 1997, p. 84. duisaient des inventions complexes du point de vue technique dans des secteurs autres que domestiques, considérés comme mineurs ${ }^{37}$. Or, elles furent désappointées de constater que la majorité des inventions brevetées étaient relatives aux domaines réservés des femmes : la maison, l'éducation. Cependant, elle souligne aussi que "Ceux qui voient " les inventions féminines " telles que les corsets comme un créneau sans grande importance commerciale devraient noter que les inventeurs masculins ont obtenu $91 \%$ des 1661 brevets de corsets pris durant le XIXe siècle $\|^{38}$. La fonction économique des domaines dits féminins est donc réelle et exploitée par les hommes.

Près de la moitié des brevets sont le fait de femmes mariées. Le régime juridique français accorde peu de droits à la femme mariée qui est passée de la tutelle de son père à celle de son mari. Ces femmes sont-elles plus autonomes ou d'un milieu plus ouvert à l'émancipation féminine? Même si nous disposions de plus d'informations (la profession par exemple), il resterait des interrogations sur le milieu culturel auquel appartient l'inventrice.

"Les femmes sont aussi les épouses et les filles des nouveaux industriels " fait justement remarquer Deborah Jaffe ${ }^{39}$. Mais qu'en est-il de leur autonomie? Ont-elles pu servir de paravent ou de prête-nom?

\section{Difficultés et limites de la recherche}

L'obligation de mise en œuvre du brevet, d'exploitation de l'invention, est inscrite dans la loi, même si cette obligation s'atténue progressivement entre 1883 et $1958^{40}$. Les inventrices ont donc dû entrer dans un processus d'innovation : produire et commercialiser leur invention pour ne pas perdre le bénéfice du brevet. Cette partie fondamentale est totalement en friche : I'INPI, consulté, ne possède pas d'informations sur le devenir des brevets. Seule une recherche dans les archives permettra de retracer le parcours d'une invention. Selon Gabriel Galvez-Behar, les revues d'inventeurs, en plein essor dans les années 1890, représentent des liens entre les inventeurs et les " capitalistes " prêts à exploiter les brevets. Elles sont un circuit de l'information techni-

37 Z. B. Khan, The democratization of invention, op. cit. note 13, p. 128. 38 " Those who view "female inventions" such as corsets as a niche area without much commercial significance should note that male inventors obtained 91 percent of the 1661 corsets patents filed during the nineteenth century I), Z. B. Khan, The democratization of invention, op. cit. note 13, p. 146.

39 " Women were also wives and daughters of the new industrialistsı, D. Jaffe, "Ingenius women", op. cit. note 12, p. 285. 40 INPI ; Yves Plasseraud, François Savignon, L'Etat et l'invention : histoire des brevets, Paris, La Documentation française, 1986, p. 81. 
que et contribuent en plus à construire le marché de l'invention ${ }^{41}$.

Outre l'étude statistique des brevets, Zorina Khan accorde, dans son livre, une grande place à l'usage qu'en ont fait les femmes : qu'elles vendent leurs droits, accordent des licences d'exploitation ou développent des entreprises pour promouvoir leurs inventions, l'auteur a retrouvé des traces de ces activités dans les " cities directories 》, soit les annuaires que l'on pourrait peut-être traduire pour la France par les documents des tribunaux du commerce. Deborah J. Warner remarque pour sa part que « en 1900, $75 \%$ des brevets accordés à des femmes durant les cinq années antérieures avaient la réputation de rapporter des retours profitables. Des inventions féminines présentées au Centennial, au moins $35 \%$ étaient en réalité produites $1{ }^{42}$

Mises à part leurs activités professionnelles qui ont grandement profité à l'économie nationale, les femmes ont certainement joué un rôle dans l'innovation en France ; il s'agit de retrouver les preuves de ce rôle.

A l'issue de cette étude, on constate l'étendue du travail à entreprendre pour rendre compte de la présence des femmes dans le domaine de l'invention en France. Seul un recensement précis des brevets et de leur devenir permettra de quantifier et

41 Gabriel Galvez-Behar, La République des inventeurs. Propriété et organisation de l'innovation en France, Rennes, PUR, 2008.

42 « By 1900, fully 75 per cent of the patents granted to women during the previous five years were reputedly yielding profitable returns. Of the inventions by women shown at the Centennial, at least 35 per cent were actually produced $॥$, D. J. Warner, "Women inventors at the Centennial », op. cit. note 15, p. 104. d'évaluer cette présence. II autorisera par ailleurs la comparaison avec les études déjà réalisées dans les pays anglo-saxons.

Il faut repérer sur une échelle plus vaste les brevets pris par des femmes et «faire parler ॥ ces brevets pour comprendre la raison et le contexte d'une activité inventrice à une époque qui ne reconnaît pas la femme comme une citoyenne, mais qui bénéficie, peut-être, de ses compétences techniques.

« [...] la pratique inventive est empreinte de rapports sociaux, depuis le statut professionnel et social de l'inventeur jusqu' au rôle des institutions. Autrement dit, l'invention n'est jamais ni gratuite ni libre ${ }^{43}$. Dans ces conditions, le contraste est fort entre l'image traditionnelle de la femme telle qu'établie dans I'histoire des femmes, entravée par son statut social, qu'elle soit bourgeoise ou ouvrière, dépendant juridiquement des hommes, et cette inventrice qui doit communiquer avec des milieux éminemment masculins. Comment dresser le portrait type de l'inventrice? Pourquoi et comment existe-t-elle ? Comment cette inventrice se situe-t-elle face à ses confrères ? Si tant est qu'on puisse dresser une échelle de valeurs des inventions, comment se situe l'inventrice par rapport à cette échelle? Toutes questions dont les réponses ne peuvent qu'apporter, à l'histoire des techniques, de celles et ceux qui les construisent, des éclairages pour mieux comprendre l'évolution d'une société.

43 Georges Ribeill, «Inventer au XIXe siècle : ingénieurs et ouvriers inventeurs au XIXe siècle $॥$, Culture technique, $\mathrm{n}^{\circ} 8$, juin 1982, pp. 217-243, p. 221. 